\title{
Traffic Estimation for Dynamic Capacity Adaptation in Load Adaptive Network Operation Regimes
}

\author{
Andreas Ahrens ${ }^{1}$, Christoph Lange ${ }^{2}$ and César Benavente-Peces ${ }^{3}$ \\ ${ }^{1}$ Hochschule Wismar, University of Technology, Business and Design, Philipp-Müller-Straße 14, 23966 Wismar, Germany \\ ${ }^{2}$ Deutsche Telekom AG, Telekom Innovation Laboratories, Winterfeldtstraße 21, 10781 Berlin, Germany \\ ${ }^{3}$ Universidad Politécnica de Madrid, Ctra. Valencia. km. 7, 28031 Madrid, Spain
}

Keywords: Traffic Prediction, Network Energy Efficiency, Wiener Filtering, Demand-aware Network Operation, Dynamic Network Dimensioning, Green Communications.

\begin{abstract}
The energy demand of telecommunication equipment and networks has been identified to be significant. In the information society such networks are vital for societal and economic welfare as well as for the people's private lives. Therefore an improved energy efficiency of telecommunication networks is essential in the context of sustainability and climate change. Load-adaptive regimes are a promising option for energy-efficient and sustainable network operation. As the capacity is adapted to temporally fluctuating traffic demands, they require a robust traffic demand estimation. As a potential solution to mitigate this problem, a method for reliable traffic demand forecasting on relevant time scales using Wiener filtering is presented. The results show that the capacity dimensioning based on the proposed Wiener filtering traffic estimation method leads to reliable outcomes enabling sustainable and efficient network operation.
\end{abstract}

\section{INTRODUCTION}

Large-scale telecommunication networks comprising a nation-wide network infrastructure often are considerable electricity consumers (Roy, 2008; Heddeghem et al., 2014). Besides their undisputed ability to support the information society in getting more environmental-friendly, for example by providing the basis for telepresence meetings - replacing in parts business trips - and remote office work - reducing commuting - they are for their selves causing environmental impact and, furthermore, the electricity consumed by the network operation affects the operators' energy bills. Therefore, improvement options have been identified throughout the recent past (e.g. (Lange et al., 2014)). Among them a prominent opportunity for improving the energy efficiency of networks is load-adaptive operation where network capacity follows traffic demands to a certain extent using different technical approaches (e.g. (Antonakopoulos et al., 2010; Francini and Stiliadis, 2010; Puype et al., 2011; Lange and Gladisch, 2011)). This is in contrast to the prevalent network design and building practice - where network capacity is above all provided based on the expected peak traffic plus a capacity reserve. Therefore load-adaptive net- work operation regimes constitute a significant step towards network energy efficiency improvements and telecommunication sector sustainability at large. Examples for such a kind of dynamic load-adaptive network operation can be found on Ethernet links (Reviriego et al., 2011), they are standardized as low power mode regimes for ADSL connections (ITUT, 2009a; ITU-T, 2009b) and furthermore they are discussed as radio access network management approaches (Ambrosy et al., 2011).

To leverage this energy efficiency improvement potential, it is essential to adapt the provided network capacity to the fluctuating traffic demands and thus, in turn, to estimate the traffic demand reliably for these capacity dimensioning purposes: The capacity of network links and sections has to be set based on sound and robust estimations of the expected traffic in particular network parts in order that network services can be provided in consistently high quality - but at improved energy efficiency.

In this contribution a method for traffic prediction is presented that makes use of Wiener filtering (Wiener, 1949; Vega and Rey, 2013; Vaseghi, 2009) as it is known from statistical signal processing: The knowledge regarding traffic behaviour from the past, e. g. from previous hours or days, is used to estimate 
the future traffic.

For this purpose, a traffic-related system model is constructed in section 2. The related Wiener filtering estimation procedure is derived in section 3 . Based on this, in section 4 a method is presented to show how the capacity of a certain network link or section can be dimensioned by using the estimated traffic function - while also taking into account a capacity reserve in a load-adaptive operation regime. The obtained results are introduced and analyzed in section 5. For verification purposes, the originally observed traffic is compared with the estimated traffic. Also, the energy consumption associated with the newly proposed capacity dimensioning strategy is calculated and compared to conventional procedures as a reference and upper boundary and to the practically unachievable case when the network capacity would follow the traffic ideally - as a second, lower, limit. The previously explained concept is verified by means of a statistical analysis where the stochastic traffic characteristics are varied and the resulting capacity dimensioning and energy efficiency is analyzed. Concluding remarks are provided in section 6 .

\section{TRAFFIC-RELATED SYSTEM MODEL}

As a basis for establishing traffic prediction algorithms real measured traffic data or a modeled traffic time function with suitable characteristics and statistics is necessary. To show the conceptual principle concept, throughout this paper a traffic model is used that is referring to an exemplary link in a network whose capacity is subject to load-adaptive switching regimes. The traffic function is constructed as follows: An underlying time function $s(k)$, with variations on a longer time scale, is used for modelling the average traffic fluctuation observed for an exemplary link as published in (DE-CIX, 2016) as shown in Fig. 1. To model the stochastic variations in the traffic on a shorter time scale, an additive white Gaussian noise $n(k)$ with zero mean and the variance $P_{\mathrm{R}}$ is added. In consequence, a fictitious but realistic traffic function $v(k)$ is obtained that is referred to an observed traffic throughout the paper. The observed (measured) traffic $v(k)$ results analytically in:

$$
v(k)=s(k)+n(k) \text {. }
$$

Fig. 2 shows exemplary curves of the observed (measured) traffic $v(k)$ and the underlying averaged traffic function $s(k)$. The resulting system model is highlighted in Fig. 3. This modelled traffic contains the long-term traffic fluctuations over a day as well as

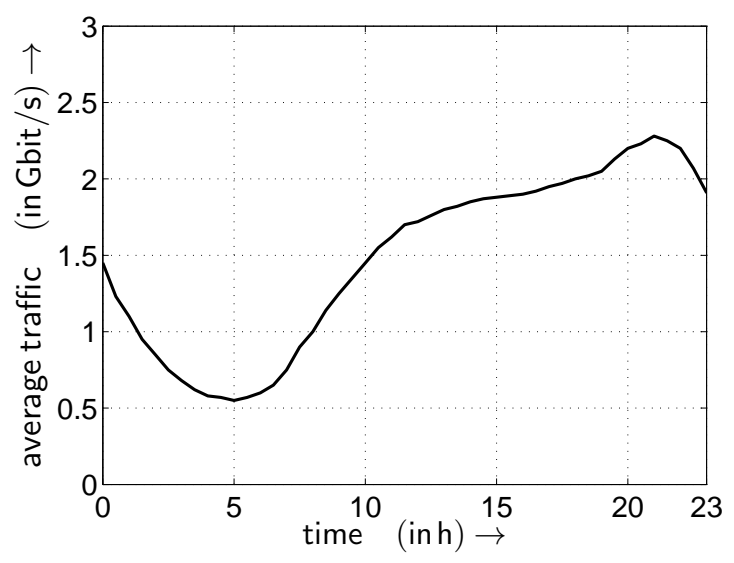

Figure 1: Average diurnal traffic time function taken from (DE-CIX, 2016).

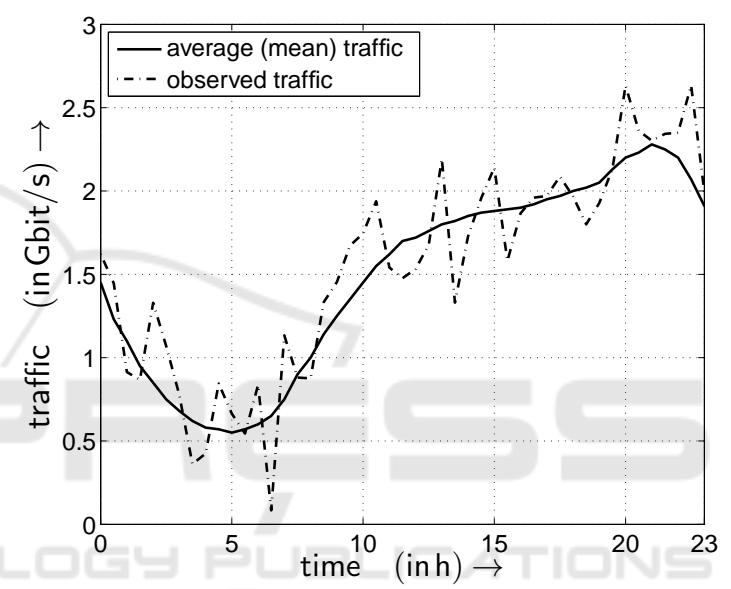

Figure 2: Characteristics of exemplarily averaged (solid line) and observed traffic (dashed line).

the inherent stochastic nature of typical broadband data traffic. The complementary cumulative distribution function (CCDF) of the modelled traffic at noon is shown in Fig. 4. Assuming a throughput of 1,72 Gbit/s (averaged traffic observed $s(k)$ ) at noon traffic fluctuations become obvious. In conclusion, in this way an appropriate traffic model has been obtained that can be described and adjusted by analytic parameters.

\section{TRAFFIC PREDICTION USING WIENER FILTERING}

Traffic prediction plays an important role when optimizing a network as the traffic demand applied to a network is a key input parameter for network design and dimensioning. The future traffic can be estimated by taking the last traffic values into account. A linear predictor can be used to estimate the traffic at the 


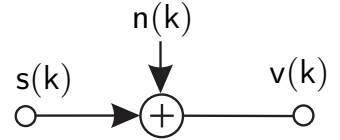

Figure 3: Resulting system model for modelling traffic fluctuations.

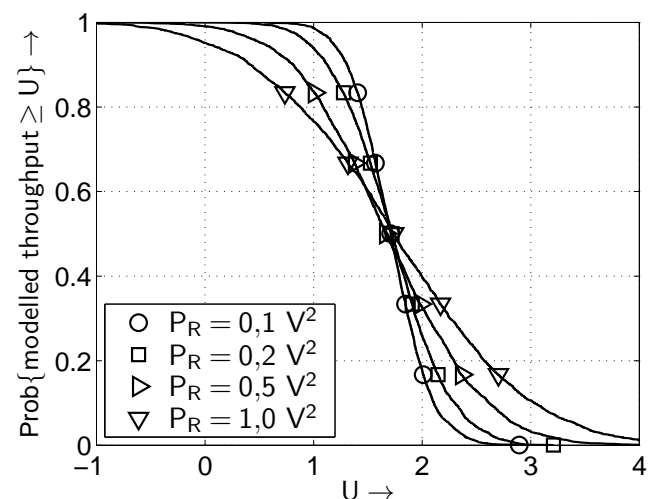

Figure 4: CCDF of the throughput at noon taking different values of the noise power $P_{\mathrm{R}}$ into account.

time $k$ by taking the last $q$ traffic values $v[k-q]$ into account and results in

$$
\hat{v}[k]=\sum_{\mu=1}^{q} p_{\mu} v[k-\mu]
$$

with the parameter $q$ describing the order of the predictor. The coefficients of the predictor $p_{\mu}$ (for $\mu=$ $1,2, \cdots, q)$ have to be defined by minimizing the energy of the error signal $e[k]=v[k]-\hat{v}[k]$, i. e.

$$
\mathrm{E}\left[(v[k]-\hat{v}[k])^{2}\right] \rightarrow \operatorname{Min}
$$

Taking (2) into account, the error signal $e[k]$ results in

$$
e[k]=v[k]-\hat{v}[k]=\sum_{\mu=0}^{q} b_{\mu} v[k-\mu] .
$$

with the parameter $b[\mu]$ defined as

$$
b[\mu]=\left\{\begin{array}{cl}
1 & \mu=0 \\
-p[\mu] & 1 \leq \mu \leq q \\
0 & \text { otherwise }
\end{array}\right.
$$

The error signal $e(k)$ appears after linear filtering of the signal $v(k)$ with the so far unknown filter coefficients $b(k)$ (see Fig. 5).

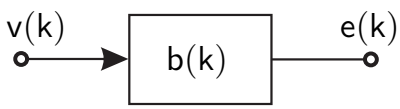

Figure 5: Error signal $e(k)$ as a function of traffic function $v(k)$.

Differentiating the error signal $e[k]$ with respect to the $b[\mu]$ (for $\mu=1,2, \ldots, q$; since $b[0]$ is assumed to be $b[0]=1)$ and setting it to zero, leads to the optimal set of filter coefficients $b[\mu]$ (for $\mu=1,2, \ldots, q$ ). Thereby, taking into consideration the squared error expression

$$
e^{2}[k]=\left(\sum_{\mu=0}^{q} b_{\mu} v[k-\mu]\right)^{2}
$$

and its partial derivatives leads to (for $\mu=1,2, \ldots, q$ )

$$
\frac{\partial e^{2}[k]}{\partial b_{\mu}}=2\left(\sum_{\mu=0}^{q} b_{\mu} v[k-\mu]\right) v[k-\mu]=0 .
$$

Taking the expectation of $e^{2}[k]$ into account, (7) can be rewritten in a matrix arrangement as

$$
\mathbf{R p}=\mathbf{r},
$$

which are also known as Yule-Walker equations. With the definition of the autocorrelation function of the real-valued signal $v(k)$ in the following form

$$
\phi_{\mathrm{vv}}[\mu]=\mathbf{E}\{v[k] v[k+\mu]\}
$$

the matrix $\mathbf{R}$ defined in (8) results in

$$
\mathbf{R}=\left(\begin{array}{cccc}
\phi_{\mathrm{vv}}[0] & \phi_{\mathrm{vv}}[-1] & \ldots & \phi_{\mathrm{vv}}[-(q-1)] \\
\phi_{\mathrm{vv}}[1] & \phi_{\mathrm{vv}}[0] & \cdots & \phi_{\mathrm{vv}}[-(q-2)] \\
\vdots & \vdots & \vdots & \vdots \\
\phi_{\mathrm{vv}}[(q-1)] & \cdots & \phi_{\mathrm{vv}}[1] & \phi_{\mathrm{vv}}[0]
\end{array}\right)
$$

Finally, the vector $\mathbf{p}$ with the unknown predictor filter coefficients as well as the vector $\mathbf{r}$ can be obtained as

$$
\mathbf{p}=\left(\begin{array}{c}
p[1] \\
p[2] \\
\vdots \\
p[q]
\end{array}\right) \quad \text { and } \quad \mathbf{r}=\left(\begin{array}{c}
\phi_{\mathrm{vv}}[1] \\
\phi_{\mathrm{vv}}[2] \\
\vdots \\
\phi_{\mathrm{vv}}[q]
\end{array}\right) \text {. }
$$

After inverting the matrix $\mathbf{R}$, the predictor coefficients $p(k)$ can be obtained.

Taking the stationary mean (averaged) traffic $s(k)$ and the added noise $n(k)$ into account, the observed noisy process $v(k)$ forms the basis for the proposed traffic prediction. Using the Wiener filter the mean square error between the estimated traffic $\hat{v}(k)$ and the mean (averaged) traffic $s(k)$ can be minimized.

In Fig. 6 the curves of the exemplary observed traffic $v(k)$ and the predicted traffic $\hat{v}(k)$ are shown: It becomes obvious that the estimated or predicted time function follows the observed traffic in tendency but is not directly useful for capacity dimensioning as there are time periods where the traffic is underestimated. Therefore, some modification or adaption of the Wiener filtering is necessary for capacity dimensioning purposes in order to take those deviations into account. The target is always a reliable network operation - meaning here sufficient capacity - and then somewhat downstream - the improved energy efficiency. 


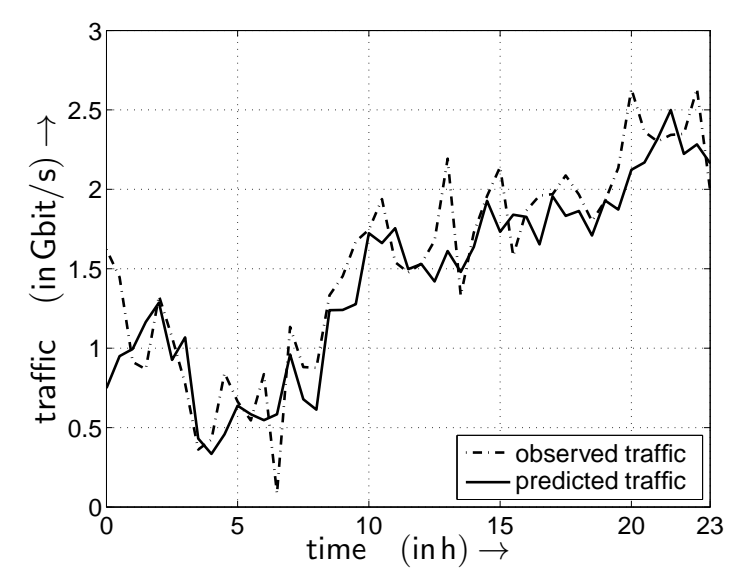

Figure 6: Observed (dashed line) and predicted (solid line) traffic time functions.

\section{CAPACITY DIMENSIONING AND ENERGY EFFICIENCY}

Provided that there is a linear dependency between capacity and power ensured by the network elements, from the capacity time function $c(t)$ a power time function $P(t)$ is obtained by

$$
P(t)=K c(t)
$$

where the factor $K$ exhibits the dimension of an energy per bit (in J/bit or Ws/bit). The actual value and magnitude of $K$ depends strongly on the system technologies and their generations. In this work the parameter $K$ is assumed to be $K=10^{-6} \mathrm{Ws} /$ bit. From the temporal power consumption curves the energy consumption during a certain period of time $T$ is obtained by

$$
E_{1}=\int_{0}^{T} P(t) \mathrm{d} t
$$

Having a capacity function which is independent from current traffic, i.e. $c(t)=c_{0}=$ const., the power consumption function $P(t)$ is assumed to be constant, i. e. $P(t)=P_{0}$. In this case the energy consumption of a considered time period $T$ is given by

$$
E_{0}=\int_{0}^{T} P_{0} \mathrm{~d} t=P_{0} T
$$

Taking into account that the power consumption function $P(t)$ follows the traffic function $v(t), P(t)$ has to be adapted according to the traffic (Fig. 7). As highlighted by Fig. 7 load adaptiveness leads to energy efficiency improvement. Now, the temporal power consumption $P(t)$ is no longer constant and the energy consumption of a considered time period $T$ is
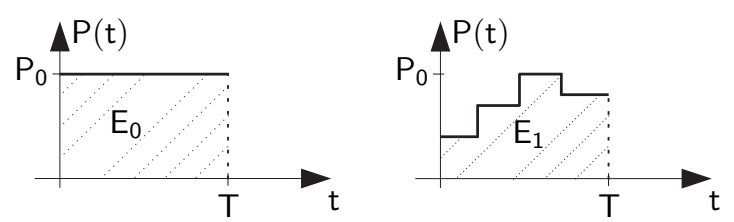

Figure 7: Energy efficiency improvement by taking power traffic-dependent time function for load-adaptiveness (right) and non load-adaptiveness (left) into account.

obtained by (13). To measure the energy efficiency improvement of particular load-adaptive case $n$, the energy efficiency parameter

$$
\varepsilon_{n}=\frac{E_{n}}{E_{0}}
$$

is used, as defined in (Lange and Gladisch, 2011). Here, $E_{0}$ describes the reference case with no loadadaptiveness at all.

\section{ENERGY EFFICIENCY RESULTS}

Based on the capacity dimensioning use cases in Fig. 8 it is assumed that the capacity follows directly the estimated traffic. As an example a noise power $P_{\mathrm{R}}=0,1 \mathrm{~V}^{2}$ is assumed. This equals $1 \mathrm{~W}$ at a linear and constant resistance of $1 \Omega$. In order to avoid a capacity bottleneck a traffic reserve $\Delta$ is added to the estimated traffic $\hat{v}(t)$, i. e.

$$
c(t)=\hat{v}(t)+\Delta
$$

to ensure a sufficient capacity. This traffic reserve $\Delta$ is especially needed for situations where the real traffic is under-estimated by the predictor.

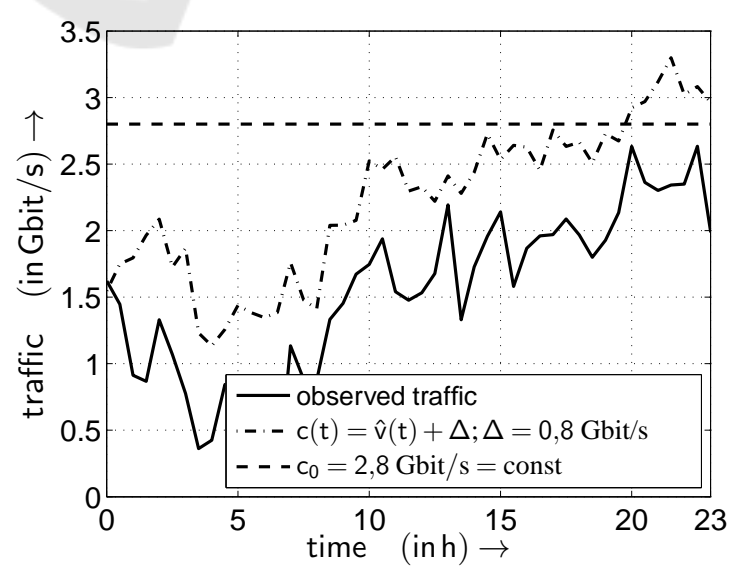

Figure 8: Capacity as a function of the estimated traffic for different parameters of the traffic reserve.

The energy efficiency of different cases of loadadaptive operation regimes is shown in Fig. 9. 
Hereby, scenario 0 describes the reference case employing no load-adaptiveness at all and scenario 1 represents the best-case limit, where the capacity follows the observed traffic ideally. Realistic load-adaptive regimes will exhibit energy efficiencies $\varepsilon_{n}$ between those boundaries. It becomes obvious, that the energy

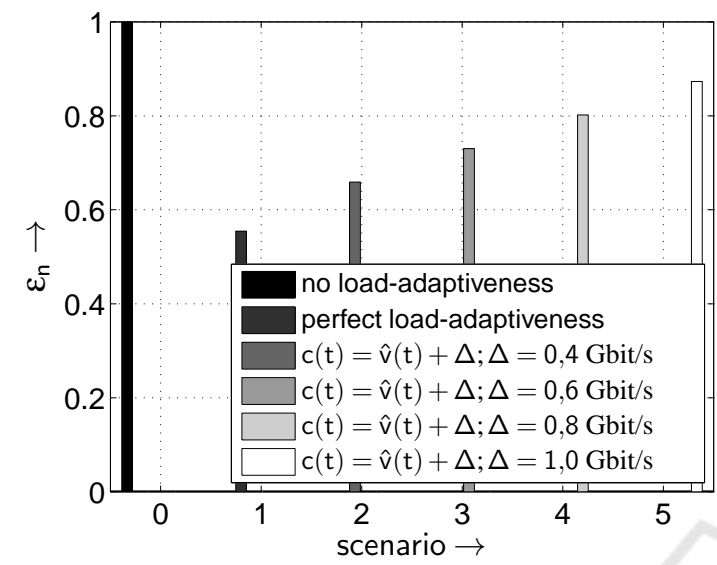

Figure 9: Energy efficiency for different parameters of the traffic reserve based on $c_{0}=2,8 \mathrm{Gbit} / \mathrm{s}$.

efficiency is increased when approximating the traffic curve more exactly. However, in scenarios where the traffic is under-estimated a capacity bottleneck could appear. The probability will doubtlessly increase for lower $\Delta$. That's why the parameter $\Delta$. has to be selected carefully.

In Fig. 10 the complementary cumulative distribution function (CCDF) is shown for the estimated traffic curve by the Wiener filtering algorithm at noon taking different levels of uncertainty (modeled by a noise power $P_{\mathrm{R}}$ ) into account. Here the probability is shown for the case that the estimated throughput is larger than a given threshold $U$. Assuming a throughput of 1,72 Gbit/s (averaged traffic observed $s(t)$ ) at noon it becomes obvious that the estimated traffic leads to a capacity bottleneck in situation where the predicted traffic is under-estimated compared to the real traffic. Only in 15 percent of all realizations the estimated traffic throughput is larger than averaged traffic observed at noon, i.e., 1,72 Gbit/s. The under estimation of the real traffic will be compensated by the introduced traffic reserve $\Delta$. It becomes obvious that the larger the uncertainty (modeled by a noise power $P_{\mathrm{R}}$ ), the higher the fluctuations in the estimated throughput.

Fig. 11 shows the estimated throughput error at noon when comparing the predicted traffic $\hat{v}(k)$ with the averaged (mean) traffic $s(k)$, i. e.

$$
\hat{v}(k)-s(k) \text {. }
$$

Taking different levels of uncertainty (modeled by the

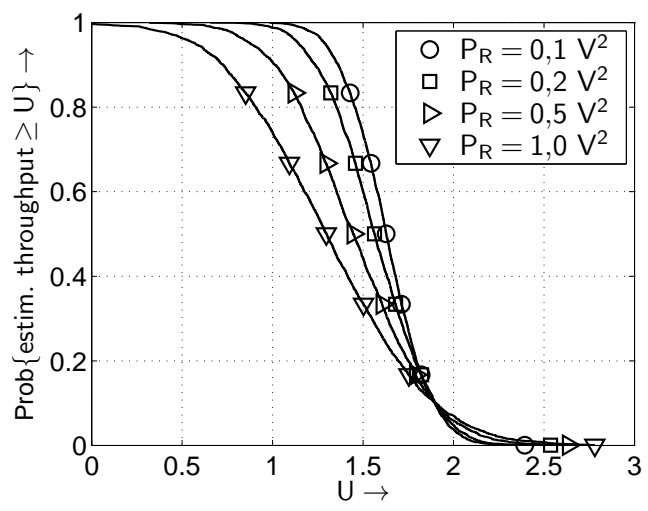

Figure 10: CCDF of the estimated throughput at noon taking different values of the noise power $P_{\mathrm{R}}$ into account.

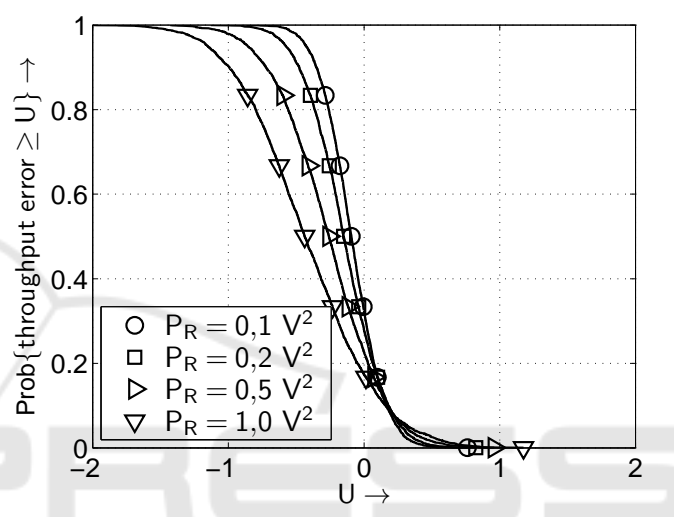

Figure 11: CCDF of the estimated throughput error at noon taking different values of the noise power $P_{\mathrm{R}}$ into account.

power $P_{\mathrm{R}}$ ) into account the importance of the introduced traffic reserve $\Delta$ is visible in situations where the traffic is underestimated.

Using the Wiener filter the mean square error between the estimated traffic $\hat{v}(k)$ and the mean (averaged) traffic $s(k)$ is minimized. Therefore, not only the noise $n(k)$ is affected by the proposed prediction algorithm but also the mean (averaged) traffic $s(k)$. Thus different slopes of the CCDF curves, depending on the levels of uncertainty, can be obtained as given in Fig. 10 and 11.

\section{CONCLUSIONS}

In the context that the energy consumption of large operator's telecommunication networks is significant, load-adaptive network operation regimes are widely accepted as a means to improve communication networks' energy efficiency. For their application to real networks, they require a reliable estimation of the expected future traffic demands on certain links and network sections in order to provide capacity accord- 
ing to the demands. A method is presented that utilizes Wiener filtering techniques to predict future traffic demands based on traffic observations from the past. The results show that this method is suitable for this kind of application in real-world network scenarios.. With the predicted traffic values and resulting capacity dimensioning significant energy efficiency improvements can be obtained under realistic preconditions. However, the results show that a sufficient reserve capacity has to be added in order to enable reliable traffic flows. As this capacity reserve itself impacts the targeted energy efficiency improvement, it has to be chosen carefully.

For application in large-scale networks such traffic prediction algorithms - as discussed and investigated throughout this article - have to be translated into robust and practically manageable software programs that converge to reliable solutions in suitable time frames. Also, a comparison to possible alternative approaches with their convergence behavior and prediction results is a task for further work.

\section{REFERENCES}

Ambrosy, A., Blume, O., Klessig, H., and Wajda, W. (2011). Energy saving Potential of Integrated Hardware and Resource Management Solutions for Wireless Base Stations. In 22nd International Symposium on Personal Indoor and Mobile Radio Communications (PIMRC), Toronto (Canada).

Antonakopoulos, S., Fortune, S., and Zhang, L. (2010). Power-aware Routing with Rate-adaptive Network Elements. In IEEE GLOBECOM, Workshop on Green Communications (GreenCom), pages 1428-1432, Miami, Florida (USA).

DE-CIX (2016). DE-CIX Traffic Statistics, http://www.decix.net/about/statistics/.

Francini, A. and Stiliadis, D. (2010). Performance Bounds of Rate-Adaptation Schemes for Energy-Efficient Routers. In International Conference on High Performance Switching and Routing (HPSR), pages 175182, Dallas, Texas (USA).

Heddeghem, W. v., Lambert, S., Lannoo, B., Colle, D., Pickavet, M., and Demeester, P. (2014). Trends in Worldwide ICT Electricity Consumption from 2007 to 2012. Computer Communications, 50:64Ü-76.

ITU-T (2009a). Recommendation ITU-T G.992.3, Asymmetric Digital Subscriber Line 2 Transceivers (ADSL2).

ITU-T (2009b). Recommendation ITU-T G.992.5, Asymmetric Digital Subscriber Line 2 Transceivers (ADSL2).

Lange, C. and Gladisch, A. (2011). Limits of Energy Efficiency Improvements by Load-Adaptive Telecommunication Network Operation. In 10th Conference of Telecommunication, Media and Internet TechnoEconomics (CTTE), pages S5-1, Berlin (Germany).

Lange, C., Kosiankowski, D., Betker, A., Simon, H., Bayer, N., v. Hugo, D., Lehmann, H., and Gladisch, A. (2014). Energy Efficiency of Load-Adaptively Operated Telecommunication Networks. IEEE/OSA Journal of Lightwave Technology, 32(4):571 - 590.

Puype, B., Vereecken, W., Colle, D., Pickavet, M., and Demeester, P. (2011). Multilayer Traffic Engineering for Energy Efficiency. Photonic Network Communications, 21(2):127-140.

Reviriego, P., Christensen, K., Rabanillo, J., and Maestro, J. A. (2011). An Initial Evaluation of Energy Efficient Ethernet. IEEE Comm. Letters, 15(5):578Ü-580.

Roy, S. N. (2008). Energy Logic: A Road Map to Reducing Energy Consumption in Telecommunications Networks. In International Telecommunications Energy Conference (INTELEC), pages paper 4-2, San Diego, California (USA).

Vaseghi, S. V. (2009). Advanced Digital Signal Processing and Noise Reduction. John Wiley \& Sons, Chichester.

Vega, L. R. and Rey, H. (2013). A Rapid Introduction to Adaptive Filtering. Springer, Heidelberg, New York.

Wiener, N. (1949). Extrapolation, Interpolation, and Smoothing of Stationary Time Series. Wiley, New York.

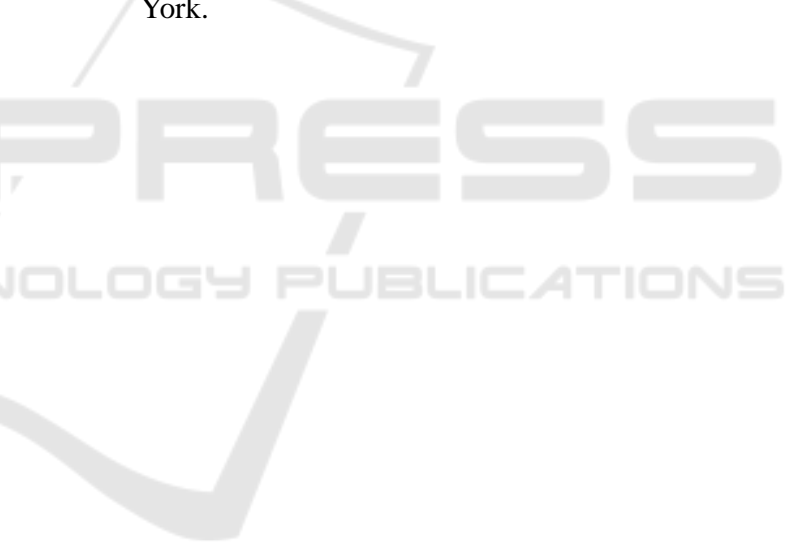

